\title{
Straddling interatrial embolus: a rare clinical dilemma
}

\author{
Elhosseyn Guella, ${ }^{1}$ Louis James Mitchell, ${ }^{2}$ Jolanta Sobolewska, ${ }^{3}$ Kamal Khan $^{2}$
}

${ }^{1}$ Department of Internal Medicine, Pennine Acute Hospitals NHS Trust, Bury, UK ${ }^{2}$ Cardiology Department, The Royal Oldham Hospital, Oldham, UK

${ }^{3}$ Department of Cardiology, Pennine Acute Hospital Trust, Oldham, UK

\section{Correspondence to} Dr Elhosseyn Guella, hguella@gmail.com

Accepted 4 June 2016
CrossMark

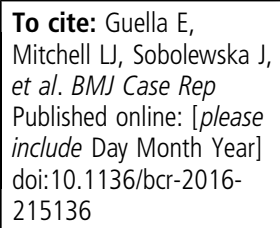

\section{SUMMARY}

We present the case of a middle-aged man who was admitted with progressive shortness of breath and haemodynamic instability. He was diagnosed as having unprovoked bilateral central pulmonary emboli.

Thrombolysis with alteplase was provided. Subsequently, an echocardiogram was performed which showed a thrombus straddling the interatrial septum. This was managed conservatively with enoxaparin anticoagulation. A repeat echocardiogram 2 days later showed complete resolution of the thrombus without clinical systemic arterial thromboembolic sequelae such as stroke, myocardial ischaemia or acute limb ischaemia.

\section{BACKGROUND}

An impending paradoxical embolism (IPDE) is a rare life-threatening condition with an estimated mortality of $16-18.4 \% .{ }^{12}$ It occurs when a venous embolus is observed straddling the interatrial septum. Treatment is aimed at decreasing morbidity and mortality caused by pulmonary and systemic arterial embolisation.

As with all rare conditions, evidence which can be used to guide treatment is limited. The little evidence which is available is observational and retrospective in nature and seems to favour surgical embolectomy as the treatment option of choice. ${ }^{1-3}$

We present the case of a completely resolving IPDE managed with anticoagulation in a patient with no comorbidities, underlying malignancy or systemic arterial emboli.

We hope this will help further our understanding of the condition and of its management.

\section{CASE PRESENTATION}

A previously fit and well 52-year-old man presented to the accident and emergency department (A\&E) reporting shortness of breath. His symptoms had started 6 months prior to hospital admission. These initially consisted of shortness of breath on exertion and mild pleuritic chest pain on deep inspiration. Basic investigations at the time, which comprised an ECG and chest X-ray, had been normal. The patient's general practitioner (GP) started a course of oral amoxicillin for a presumed lower respiratory tract infection.

The dyspnoea relentlessly progressed to shortness of breath at rest over the ensuing 6 month period. This prompted the patient to revisit his GP who sent him straight to the local A\&E.

On presentation, he was found to be tachycardic (100 bpm), hypotensive $(88 / 50 \mathrm{~mm} \mathrm{Hg})$, tachypnoeic (30 breaths per minute) and hypoxic (oxygen saturations of $89 \%$ on room air). He reported that his breathlessness was associated with an unintended weight loss of more than $10 \mathrm{~kg}$. There was no associated lymphadenopathy, fevers, night sweats or cough. He did not have any further chest pain since his initial presentation to his GP 6 months previously. He also denied having any leg swelling, calf tenderness or any prolonged period of immobility.

He had no family history of thromboembolic, cardiovascular or other hereditary disease.

A chest X-ray was performed which was normal. ECG showed non-dynamic $T$ wave inversion in leads V1-V3.

His D-dimer numeric was elevated at $3137 \mathrm{ng} / \mathrm{mL}$. Troponin levels were not measured during the admission. A working diagnosis of pulmonary embolism (PE) was made and the Pulmonary Embolism Severity Index (PESI) was calculated to be 132, which put the patient in a very high-risk category. Enoxaparin was given subcutaneously at a dose of $1.5 \mathrm{mg} / \mathrm{kg}$ within $3 \mathrm{~h}$ of presentation. This was promptly followed by CT pulmonary angiography (CTPA) which confirmed the presence of extensive bilateral central pulmonary emboli (PE) (figure 1A-D). No definite intracardiac thrombi or masses were detected (figure 2A, B). The patient was transferred to the high dependency unit (HDU). It was decided to provide primary reperfusion treatment in the form of systemic thrombolysis. Thrombolysis with alteplase was started $1 \mathrm{~h}$ after the administration of enoxaparin. This was given as a bolus of $10 \mathrm{mg}$ over $2 \mathrm{~min}$ which was followed by $77 \mathrm{mg}$ over a $2 \mathrm{~h}$ period.

The patient improved clinically and haemodynamically by completion of thrombolysis. His heart rate decreased to $85 \mathrm{bpm}$, blood pressure increased to $110 / 70 \mathrm{~mm} \mathrm{Hg}$ and respiratory rate decreased to 21 breaths per minute. He was still requiring a $15 \mathrm{~L}$ non-rebreather mask to achieve oxygen saturations of $95 \%$.

\section{INVESTIGATIONS}

CT of the abdomen and pelvis with contrast did not reveal any underlying malignancy. A thrombophilia screen was performed. Factor V Leiden mutation and prothrombin gene variant were not detected. The functional antithrombin III, protein $\mathrm{C}$ and protein $\mathrm{S}$ levels were within the normal range. Urine immunifixation did not show any Bence Jones proteins. Thyroid stimulating hormone levels had been checked 3 months previously and were normal $(0.93 \mu / \mathrm{L})$.

Venous duplex examination of the lower limbs was not performed as it was not felt it would change management. 

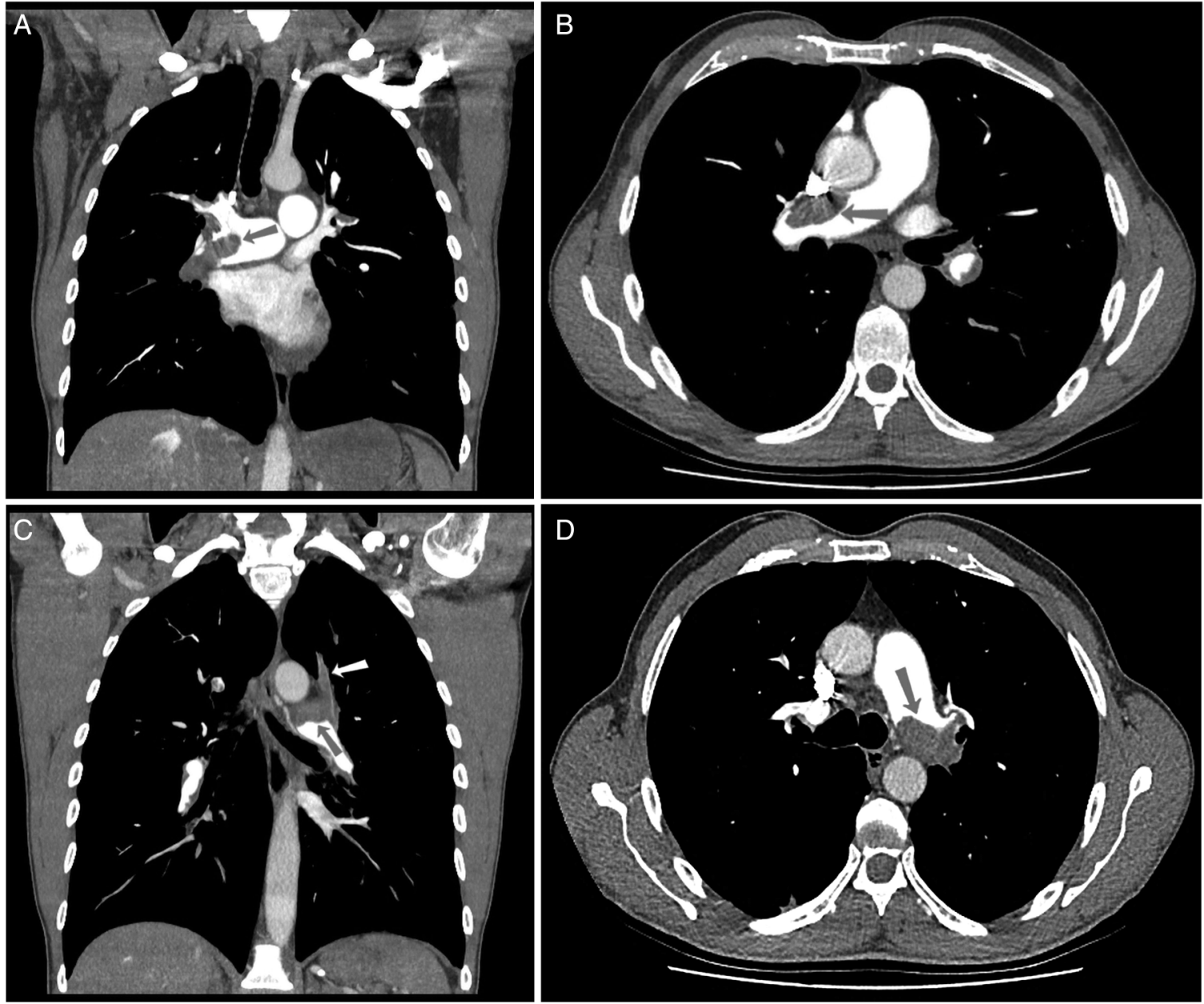

Figure 1 (A) Coronal CT section showing a massive right pulmonary artery saddle embolus (arrow). The larger part can be seen extending into the right lower lobe branch and the smaller fragment can be seen extending into the right upper lobe branch. (B) Axial CT section showing a massive right pulmonary artery embolus (arrow). (C) Coronal CT section showing a left main pulmonary artery embolus (red arrow). The embolus can be seen extending into the left upper lobe artery (white arrow). (D) Axial CT section showing a left main pulmonary artery saddle embolus (arrow).

A bedside transthoracic echocardiogram (TTE) was performed 3 days after thrombolysis for the quantification of the right ventricular (RV) function. As expected, it showed dilation of the right-sided chambers, RV dysfunction, severe tricuspid regurgitation and severe pulmonary hypertension.

Rather unexpectedly, a large mobile serpentine mass was found to be straddling the interatrial septum (figure 3A, B and video 1). There were no previous echocardiograms for comparison.

\section{DIFFERENTIAL DIAGNOSIS}

The differential diagnosis of atrial masses generally includes thrombi, tumours and vegetations (which can typically be visualised arising from valves in the context of infective endocarditis).

Cardiac tumours can either be primary or secondary. Primary cardiac tumours are extremely rare and include benign tumours such as atrial myxomas, rhabdomyomas, lipomas, papillary fibroelastomas, haemangiomas and fibromas as well as malignant
Figure 2 (A) Coronal CT section of the heart and great vessels. The superior vena cava, inferior vena cava, right atrium and ascending aorta are devoid of emboli. (B) Axial CT section of the heart. The right atrium (RA) and ventricle (RV) are devoid of emboli.
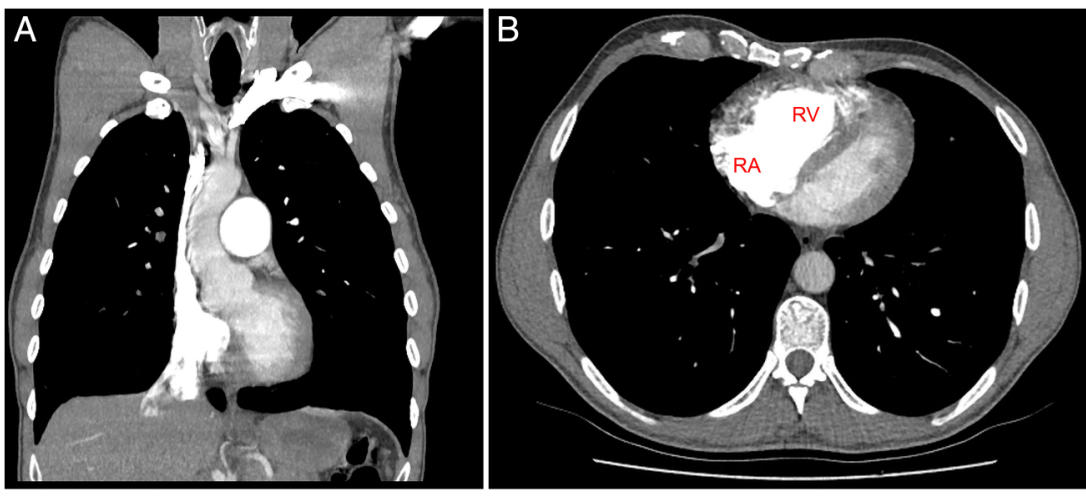
Figure 3 (A) Transthoracic echocardiogram showing a straddling embolus (arrow) extending from the inter-atrial septum into a dilated right atrium. (B) Transthoracic echocardiogram during early systole showing a straddling embolus crossing the inter-atrial septum from the left atrium to the right atrium. The embolus can be seen extending through the mitral valve (red arrow).
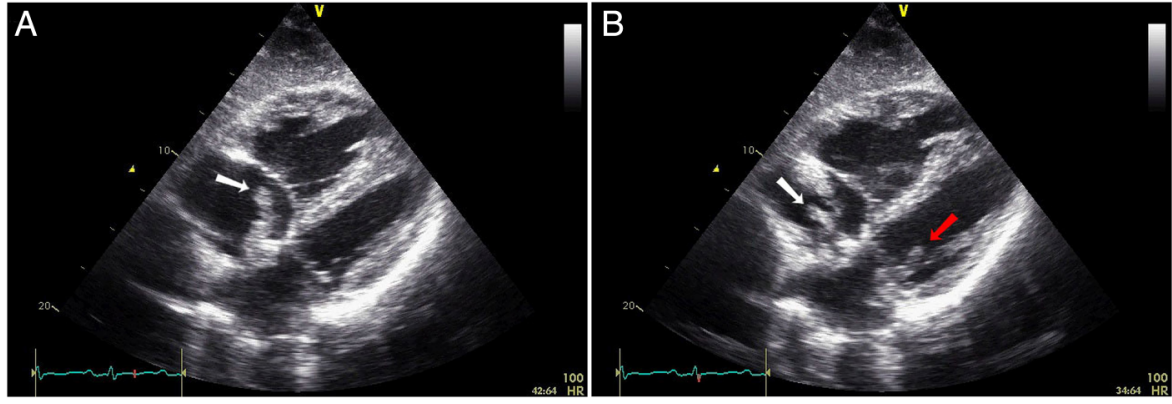

tumours such as sarcomas and primary cardiac lymphomas. Secondary tumours are much more prevalent than primary tumours and often arise from primaries such as lung cancer, breast cancer, malignant melanoma and renal cell carcinoma. The normal contrast CT of the abdomen and pelvis pointed away from such malignant causes.

The fact that the mass was biatrial, showed striking mobility and extended from the interatrial septum helped narrow down the differential diagnosis substantially. The main diagnostic challenge in this context was differentiating between impending paradoxical emboli (IPDE) and biatrial myxoma. ${ }^{4}$

Echocardiographic features which would be suggestive of a myxoma include a heterogeneous echotexture, the presence of calcification or evidence of cavitation. None of these features were present in our case. Conversely, the echocardiogram exhibited a number of features which were highly suggestive of an IPDE. Such features included the fact that the mass had a shape that changed during the different stages of the cardiac cycle, was of a homogeneous echotexture and had a typical serpentine morphology. ${ }^{1}$

The typical echocardiographic findings as well as the clinical picture in view of the presence of concomitant pulmonary emboli and a favourable pressure gradient supported the diagnosis of an IPDE.

\section{TREATMENT}

At this stage, the treatment options for the IPDE included thrombolysis, transcatheter embolectomy, surgical embolectomy or continued anticoagulation. We discussed the case with two separate tertiary cardiothoracic centres with a view to transfer for surgical embolectomy. Two separate issues were discussed with each of the cardiothoracic centres. These issues were first the optimal management of the IPDE and second that of the residual

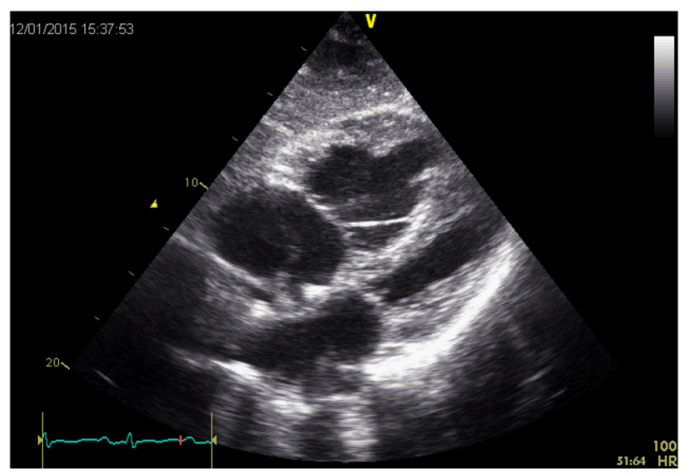

Video 1 Transthoracic echocardiogram showing a serpentine embolus extending from the left atrium to a dilated right atrium. The embolus can be seen extending through the mitral valve. pulmonary emboli. With regard to the IPDE, one centre recommended thrombolysis while the other recommended continued anticoagulation. Neither centre recommended transfer for surgical embolectomy and patent foramen ovale (PFO) closure. Both centres advised that pulmonary thrombectomy was not required immediately, citing a number of reasons. First, the patient had stabilised from a haemodynamic standpoint following thrombolysis 3 days previously. Second, it was felt that the history (which spanned over a 6-month period) and the presence of pulmonary hypertension were suggestive of some degree of chronicity. Finally, it was pointed out that there was already evidence of clots in the small and medium sized arteries.

We then proceeded to discuss the case with the regional interventional cardiology team which advised against catheter-based IPDE embolectomy for fear of thrombus fragmentation.

It was decided to persevere with enoxaparin anticoagulation.

A TTE performed 2 days later showed complete resolution of the interatrial thrombus (figure 4 and video 2).

This thrombus resolution occurred without any signs or symptoms suggestive of systemic arterial embolisation such as transient ischaemic attacks (TIA), stroke, myocardial, gut or limb ischaemia.

Furthermore, there was no deterioration in breathlessness or oxygen saturations to suggest further clinically significant pulmonary embolisation.

A repeat CTPA performed 11 days after thrombolysis showed reduction in the volume of the pulmonary emboli in the right and left pulmonary arteries and in subsegmental branches bilaterally.

\section{OUTCOME AND FOLLOW-UP}

The patient was discharged home on warfarin after successfully weaning off oxygen over a 2 -week period.

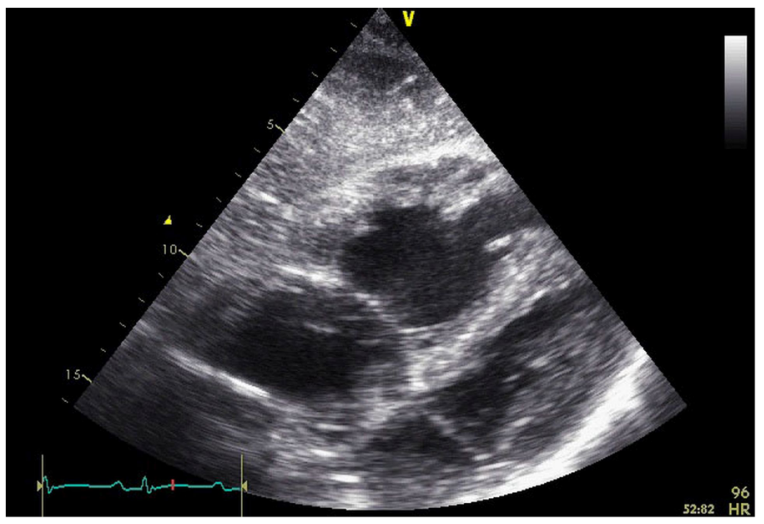

Figure 4 Transthoracic echocardiogram showing complete resolution of the embolus. 


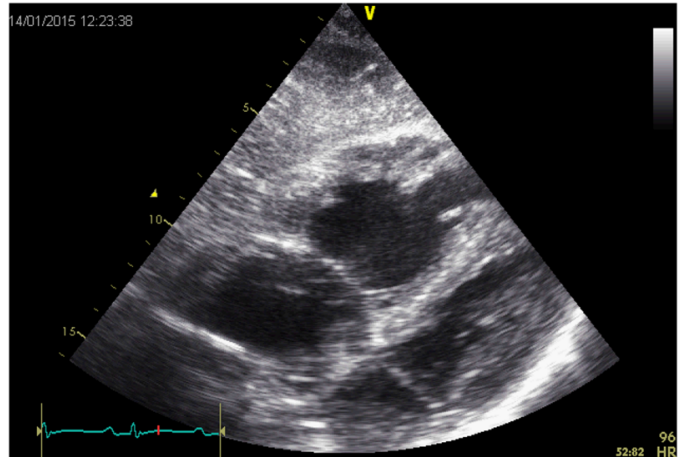

Video 2 Transthoracic echocardiogram showing complete resolution of the embolus.

A repeat echocardiogram at 3 months showed persistent pulmonary hypertension with a RV systolic pressure (RVSP) of $55 \mathrm{~mm} \mathrm{Hg}$. It also showed that there was a minimal spontaneous left to right jet through the atrial septum which could only be detected on a four chamber view (figure 5 and video 3 ). There was no echocardiographic evidence of an atrial septal defect (ASD). This confirmed that the IPDE had formed across a PFO.

The patient remained significantly breathless with an NYHA class of III.

He was referred to the national pulmonary hypertension centre for specialised treatment of his chronic thromboembolic pulmonary hypertension and consideration of surgical thromboendarterectomy.

\section{DISCUSSION}

The phenomenon of paradoxical embolisation has been recognised for a long time with the diagnosis having been made on clinical grounds as early as $1877 . .^{5}$ Otherwise, before the advent of echocardiography, straddling interatrial emboli were often only detected on postmortem examination. ${ }^{56}$

The diagnosis of IPDE was first made by Nellessen et $\mathrm{al}^{7}$ in 1985 with the use of transoesophageal echocardiography (TOE). Since then, the diagnosis has been made with increasing frequency with the use of TOE and TTE.

IPDEs are most often observed passing through PFO, as in our case, though cases of transit through ASDs have been reported. ${ }^{2}$

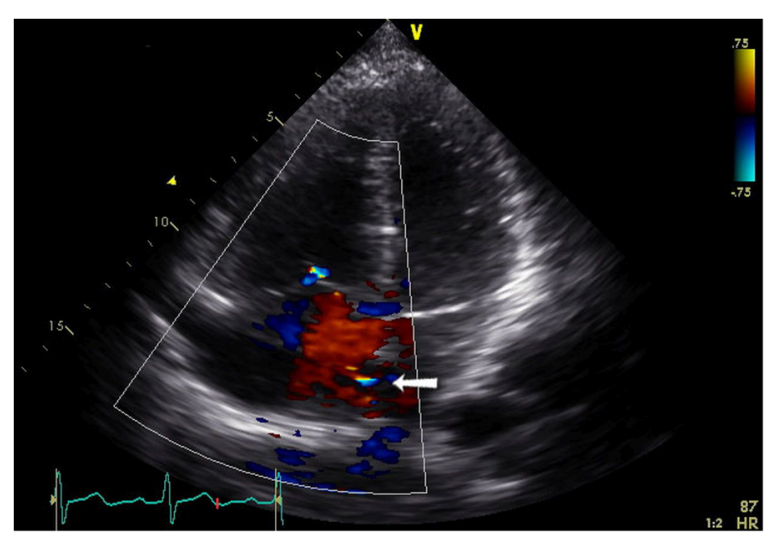

Figure 5 Transthoracic echocardiogram four-chamber view revealing a minimal spontaneous left to right jet through the atrial septum which is suggestive of a small patent foramen ovale.

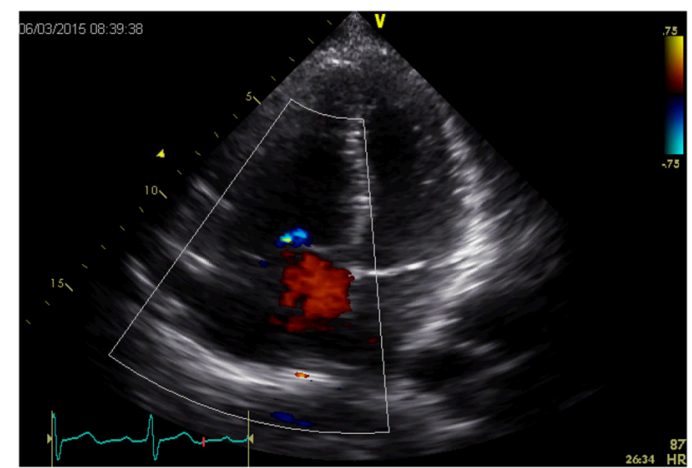

Video 3 Transthoracic echocardiogram four-chamber view revealing a minimal spontaneous left to right jet through the atrial septum which is suggestive of a small patent foramen ovale.

IPDE can be associated with stroke, ${ }^{8}$ transient ischaemic attacks, ${ }^{9}$ myocardial infarction, ${ }^{9}{ }^{10}$ gut ischaemia, renal infarcts ${ }^{11}$ and ischaemic limbs. ${ }^{12}$ It is clear, however, that systemic arterial embolisation is not invariable in the context of IPDE, as can be noted here.

IPDE is associated with high mortality (16-18.4\%). ${ }^{1}{ }^{2}$ Most deaths occur within $24 \mathrm{~h}$ of diagnosis with the major causes of death being cardiogenic shock (61\%) and stroke (39\%). ${ }^{2}$

The optimal management of IPDE is controversial. Strategies that have been used in the management of IPDE are surgical embolectomy, ${ }^{13}$ thrombolysis (including modified thrombolysis regimens) ${ }^{14}$ and anticoagulation. ${ }^{15}$ Transcatheter embolectomy has never been performed in this context due to concerns regarding embolus fragmentation.

Surgical embolectomy with foramen ovale closure is widely considered to be the first-line treatment for IPDE. ${ }^{1-3}$ Retrospective observational studies have shown a statistically significant decrease in systemic embolisation when compared with anticoagulation. Mortality also tended to be lower, but this decreased mortality was not found to be statistically significant. ${ }^{2}$

Anticoagulation is considered to be a second-line treatment and tends to be used in older patients and those with multiple comorbidities. Thrombolysis, on the other hand, tends to be used in high-risk patients such as those with haemodynamic compromise. ${ }^{1}$ It is associated with the highest rate of arterial embolisation after commencement of treatment when compared with other treatment modalities. It is also associated with a non-statistically significant increase in mortality when compared with surgery. ${ }^{2}$ It is not clear whether this points to the inferiority of thrombolysis in this context or simply reflects the fact that patients treated with thrombolysis tended to be sicker.

All the above findings must be interpreted with caution as the level of evidence is weak and the potential bias is great.

Our patient's admission CTPA had not revealed the presence of any definite intra-atrial thrombi. This suggests that the IPDE was either non-existent on admission or that it was too small and mobile to be detected with CT. In either case, it would not seem that thrombolysis could have led to the resolution of the IPDE as alteplase has a very short half-life of $5 \mathrm{~min}$ and was administered 3 days prior to the detection of the IPDE.

Our patient's thrombus resolution is not in itself surprising. A similar outcome has been almost invariably demonstrated in cases where follow-up echocardiography was performed after treatment with heparin. ${ }^{1}$ What is surprising is the speed with which the thrombus dissolved. Previously reported cases had required between 4 days and 6 weeks for thrombus resolution. ${ }^{1}$ 


\section{Patient's perspective}

- I was admitted to The Royal Oldham Hospital (first to high dependency unit and then to coronary care unit) suffering with severe breathing difficulties and weight loss. After being put on oxygen and undergoing a CT scan and other tests, it was discovered I had suffered an acute pulmonary embolism.

- The consultant in charge at the time, Dr. Kamal Khan, contacted surgical centres at Wythenshaw and Papworth hospital and decided between them to carry on with medical management. A few day later, an echocardiogram revealed the thrombus was no longer present.

- After close monitoring and more tests, I was discharged from hospital. Although still unsteady on my feet, my general health had vastly improved.

- Both I and my family are under no doubt that the doctors and nurses had saved my life with their quick reactions to the condition and we remain eternally grateful to them.

- At this moment in time, I am under Sheffield Hallamshire Hospital and have been diagnosed with chronic thromboembolic pulmonary hypertension and am being referred to Papworth Hospital for surgery to remove the clots from my pulmonary arteries.

- Being a fit and healthy person who worked out three times a week, never smoked and only enjoyed the occasional drink, it is hard for me to understand where these clots have come from.

\section{Learning points}

- Impending paradoxical embolism (IPDE) is an extremely rare condition where emboli are found straddling the interatrial septum.

- Increased right atrial pressure (usually in the context of pulmonary embolism) predisposes to paradoxical embolisation.

- Surgical embolectomy with patent foramen ovale closure is currently the first-line treatment in patients with IPDE who are good surgical candidates. This is based on retrospective data showing a statistically significant decrease in systemic embolisation.

- Medical management of IPDE in the form of anticoagulation may be a viable alternative to surgical embolectomy.

Regarding our management of the massive PE, we followed the '2014 ESC Guidelines on the diagnosis and management of acute pulmonary embolism' by providing first-line primary reperfusion treatment in the form of systemic thrombolysis. ${ }^{16}$ This was associated with marked improvement in the patient's haemodynamic status. Should systemic thrombolysis have been contraindicated or unsuccessful in improving the patient's haemodynamic status, we would have considered transferring the patient for surgical embolectomy, catheter-based thrombectomy or catheter-directed thrombolysis as advocated by the guidelines.

In terms of our choice of formal anticoagulation, we followed our local hospital policy by starting warfarin. The use of a new oral anticoagulant (NOAC) would also have been a suitable option.

Our case demonstrates the fact that low molecular weight heparin has been used successfully in the management of IPDE. Having said this, surgical embolectomy with PFO closure remains the first-line treatment for this rare condition and should be considered in all cases.

Acknowledgements The authors would like to thank Dr Ramaswany Ravichandran (consultant radiologist) for his expert input regarding the CT images. The authors would also like to thank Dr Lina Darwich for her help with image illustration.

Contributors EG performed a literature search, obtained consent from the patient and wrote the case report. LJM reviewed the manuscript prior to submission. JS provided the echocardiography images and reviewed the manuscript prior to submission. KK identified the case as being suitable for publication and reviewed the manuscript prior to submission. All authors were involved in the patient's care.

Competing interests None declared.

Patient consent Obtained.

Provenance and peer review Not commissioned; externally peer reviewed.

\section{REFERENCES}

1 Fauveau $\mathrm{E}$, Cohen A, Bonnet $\mathrm{N}$, et al. Surgical or medical treatment for thrombus straddling the patent foramen ovale: impending paradoxical embolism? Report of four clinical cases and literature review. Arch Cardiovasc Dis 2008;101:637-44.

2 Myers $\mathrm{P}$, Bounameaux $\mathrm{H}$, Panos $\mathrm{A}$, et al. Impending paradoxical embolism. Chest 2010;137:164-70.

3 Aboyans V, Lacroix P, Ostyn E, et al. Diagnosis and management of entrapped embolus through a patent foramen ovale. Eur J Cardiothoracic Surg 1998;14:624-8.

4 Meacham R, Headley A, Bronze M et al. Impending paradoxical embolism. Arch Intern Med 1998;158:438.

5 Johnson B. Paradoxical embolism. J Clin Pathol 1951;4:316-32.

6 Corrin B. Paradoxical embolism. Heart 1964;26:549-53.

7 Nellessen U, Daniel WG, Matheis G, et al. Impending paradoxical embolism from atrial thrombus: correct diagnosis by transesophageal echocardiography and prevention by surgery. J Am Coll Cardiol 1985;5:1002-4.

8 Faustino A, Costa G, Providencia $R$, et al. Impending paradoxical embolism with a thrombus crossing a patent foramen ovale. Case Reports 2012;2012:bcr2012006662.

9 Willis $\mathrm{S}$, Welch T, Bartoszek $\mathrm{M}$, et al. Impending paradoxical embolism presenting as a pulmonary embolism, transient ischemic attack, and myocardial infarction. Chest 2007;132:1358-60.

10 Dietz D, Cleveland J, Chewning K, et al. Impending paradoxical embolism presenting as myocardial infarction. J Cardiol Cases 2013;7:e145-8.

11 Mintz R, Brody K. Impending paradoxical embolism: a case report. J Emerg Med 2013;45:19-21.

12 Egred M, Patel J, Walton S. Impending paradoxical embolism. Circulation 2001;103: e113-14.

13 Podroužková $\mathrm{H}$, Horváth V, Hlinomaz $\mathrm{O}$, et al. Embolus entrapped in patent foramen ovale: impending paradoxical embolism. Ann Thorac Surg 2014;98:e151-2.

14 Turfan M, Vatankulu M, Murat S, et al. Thrombolytic treatment of simultaneous pulmonary embolism and impending paradoxical embolism through a patent foramen ovale: a different thrombolytic regimen. Heart Lung Circ 2012;21:225-8.

15 Provenzal A, Blanco P, Nomura J. Impending paradoxical embolus. J Emerg Med 2015;48:466-7

16 Konstantinides SV, Torbicki A, Agnelli G, et al., Task Force for the Diagnosis and Management of Acute Pulmonary Embolism of the European Society of Cardiology (ESC). 2014 ESC Guidelines on the diagnosis and management of acute pulmonary embolism. Eur Heart J 2014;35:3033-69, 3069a-3069k. 
Copyright 2016 BMJ Publishing Group. All rights reserved. For permission to reuse any of this content visit http://group.bmj.com/group/rights-licensing/permissions.

BMJ Case Report Fellows may re-use this article for personal use and teaching without any further permission.

Become a Fellow of BMJ Case Reports today and you can:

- Submit as many cases as you like

- Enjoy fast sympathetic peer review and rapid publication of accepted articles

- Access all the published articles

- Re-use any of the published material for personal use and teaching without further permission

For information on Institutional Fellowships contact consortiasales@bmjgroup.com

Visit casereports.bmj.com for more articles like this and to become a Fellow 